\title{
Qual o efeito do tratamento na semente contra infecção de Bipolaris sorokiniana na fase vegetativa da cevada?
}

\author{
What is the effect of seed treatment against Bipolaris sorokiniana infection in barley during \\ the vegetative stage?
}

\author{
Keilor da Rosa Dorneles*, Anderson Eduardo Brunetto, André Alves, Leandro José Dallagnol \\ Universidade Federal de Pelotas, Pelotas, RS, Brasil. *Autor para correspondência: keilor.rd@hotmail.com
}

Submissão: 11/04/2020 | Aceite: 22/06/2020

\begin{abstract}
RESUMO
O objetivo da pesquisa foi avaliar o efeito residual do tratamento das sementes de cevada com fungicida $e$ fertilizante silicatado no controle da mancha marrom no estágio vegetativo da planta. $O$ delineamento experimental utilizado foi inteiramente casualizado em esquema bifatorial $4 \times 3$, constituído por quatro tratamento de sementes (TS): testemunha (água destilada), fungicida (triadimenol), fertilizante silicatado e a mistura fungicida + fertilizante silicatado e plantas com 30, 45 e 60 dias após a emergência (DAE). Para avaliar o efeito residual (TS), as plantas foram inoculadas com uma suspensão de esporos de Bipolaris sorokiniana. A avaliação da severidade ao longo do tempo foi utilizada para calcular a área abaixo da curva de progresso da mancha marrom (AACPMM). Aos $30 \mathrm{DAE}, \mathrm{o}$ tratamento com fungicida foi o mais eficiente com a redução de $64 \%$ na AACPMM, seguido do fertilizante silicatado com $56 \%$. A mistura também reduziu a AACPMM, mas com eficiência inferior aos produtos isolados.
\end{abstract}

PALAVRAS-CHAVE: Hordeum vulgar, efeito residual, fungicida sistêmico, mancha marrom, silício.

\begin{abstract}
The objective of the research was to evaluate the residual effect of the seeds treatment with fungicide and silicate fertilizer in the control of brown spot in the vegetative stage of the barley plant. The experiment was a two-way factorial with four seed treatments (ST): control (distilled water), fungicide (triadimenol), silicate fertilizer and the fungicide + silicate fertilizer and three periods of 30,45 and 60 days after emergence (DAE). Barley plants were inoculated with a spore suspension of Bipolaris sorokiniana. The disease severity assessment over time was used to calculate the area under the brown spot progress curve (AUCPBS). At 30 DAE, fungicide treatment was the most efficient with reduction of $64 \%$ in the AUCPBS, followed by silicate fertilizer with $56 \%$. The mixture also reduced AUCPBS, but with lower efficiency than isolated products.
\end{abstract}

KEYWORDS: Hordeum vulgare, brown spot, residual effect, systemic fungicide, silicon.

A maioria das culturas agrícolas, utilizadas para alimentação humana e animal, são propagadas por sementes (SAATH \& FACHINELLO 2018). Dentre essas, inúmeras são consideradas de importância primordial, como: soja, milho, trigo, arroz, feijão e a cevada. Todavia, essas culturas compartilham limitantes/desafios em comum. Todas possuem as suas sementes afetadas por patógenos, os quais interferem negativamente na germinação e desenvolvimento inicial da plântula, além ser uma via de disseminação do patógeno para áreas indenes (BRASIL 2009).

Assim, como medida de proteção se utiliza o tratamento de sementes. Prática, que no sentido abrangente, significa à aplicação de processos e substâncias que preservam ou aperfeiçoam o desempenho das sementes, permitindo a expressão máxima do seu potencial genético (LEMES et al. 2019). De maneira majoritária, são aplicados fungicidas que protegem a semente, desde o contato inicial com o solo até o início do crescimento da planta (PINTO 2000, CAMILO et al. 2017).

Portanto, doenças que ocorrem na parte aérea da planta, como a mancha-marrom causada por Bipolaris sorokiniana (Sacc.) Shoemaker na cevada, pode vir a ser manejada através do tratamento das sementes com produtos sistêmicos. Já que os programas de manejo químico em parte aérea, passam a ser aplicados apenas a partir dos 30 dias após a emergência da planta, a qual permanece por esse período 
vulnerável ao patógeno presente no ambiente. Em trigo, a severidade do oídio e da ferrugem-da-folha foi reduzida nos primeiros dias do crescimento vegetativo através do tratamento de sementes com fungicida do grupo químico triazol (GOULART 1999). Além do uso de fungicidas, a aplicação de produtos silicatos nas sementes, também tem proporcionado ação fisiológica nas plantas, promovendo um crescimento mais vigoroso das mesmas, como visto em arroz (TUNES et al. 2014, MACHADO \& QUEIROZ 2018).

Contudo, mesmo com o conhecimento da influência do tratamento da semente sobre a fase vegetativa da planta. Informações sobre a duração ou persistência da ação residual do produto aplicado, ou seus efeitos, ainda são restritas, principalmente em cevada. Diante do exposto, o objetivo com essa pesquisa foi avaliar o efeito residual do tratamento das sementes de cevada, com fungicida e fertilizante silicatado, no controle da mancha-marrom no estágio vegetativo da planta.

Sementes de cevada da cultivar BRS Caue (Embrapa $\left.{ }^{\circledR}\right)$ foram utilizadas no experimento. A semeadura foi realizada em vasos plásticos com capacidade de quatro litros, contendo solo, o qual teve sua fertilidade previamente corrigida, de acordo com as recomendações técnicas para a cultura da cevada.

$O$ experimento foi organizado em delineamento inteiramente casualizado, em esquema bifatorial $4 \times$ 3 , constituído por quatro tratamento de sementes e exposição ao patógeno aos 30, 45 e 60 dias após a emergência. Cada tratamento foi composto por cinco repetições.

Os tratamentos utilizados foram: testemunha (água destilada), fungicida (triadimenol, $150 \mathrm{~g} \mathrm{~L}^{-1}$ ), fertilizante silicatado (composição: P 5,7\% + K 5,2\% + Co 1\% + Mo $2 \%+\mathrm{Ca} 6 \%+\mathrm{Mg} 4,4 \%+\mathrm{Zn} 2 \%+\mathrm{Fe}$ $4 \%$ e silício $68,1 \%, 5 \mathrm{~g} \mathrm{~kg}^{-1}$ de semente) e a mistura fungicida + fertilizante silicatado. O recobrimento das sementes ocorreu manualmente, adicionando o produto dissolvido em água, na dose recomendada pelo fabricante, em sacolas plásticas transparentes, com capacidade para 3 litros, agitando continuamente as sementes até garantir adequada homogeneização do tratamento. Em seguida, as sementes foram postas em repouso por uma hora e posteriormente semeadas.

Para avaliar o efeito residual dos tratamentos de sementes (TS), plantas com 30, 45 e 60 dias após a emergência foram inoculadas com $B$. sorokiniana. A inoculação foi por meio da pulverização da suspensão de esporos, ajustada para $1 \times 10^{4}$ conídios $\mathrm{mL}^{-1}$, na superfície das folhas com auxílio de um borrifador manual. Toda a superfície foliar de todas as plantas foram completamente cobertas pela pulverização, contudo sem provocar escorrimento superficial. Após a inoculação, as plantas foram mantidas por $48 \mathrm{~h}$ em câmara de nevoeiro com umidade relativa superior a $90 \%$ e temperatura $22 \pm 3{ }^{\circ} \mathrm{C}$. Logo após esse período, as plantas foram mantidas em casa de vegetação, com umidade relativa do ar de $55 \%$ e temperatura de 22 $\pm 3^{\circ} \mathrm{C}$.

A avaliação da severidade da doença foi realizada aos 3,5, 9, 11, 13 e 16 dias após a inoculação (DAl), sendo estimada em porcentagem, com base na proporção da área das folhas afetadas pela doença em relação a área foliar total da planta. A partir dos dados da severidade foi calculada a área abaixo da curva de progresso da mancha-marrom (AACPMM) (MENEGON et al. 2005).

Para evidenciar a homogeneidade dos dados foi aplicado o teste de Shapiro Wilk, para todas as variáveis. Os dados dos dois experimentos foram unidos e submetidos à análise de variância (ANOVA) e as médias dos tratamentos comparadas pelo teste de Tukey $(p \leq 0,05)$.

Aos 30 dias após emergência, o tratamento de sementes com fungicida reduziu a AACPMM em 64\%, seguido do fertilizante silicatado com $56 \%$ e da mistura fungicida + fertilizante com $34 \%$, quando comparado com o controle (Figura 1 e Figura 2).

Entretanto, a partir de 45 dias após a sua aplicação na semente os tratamentos não interfiram no desenvolvimento da mancha-marrom (Figura 1). A AACPMM é uma variável elementar, pois sumariza a epidemia como um todo, ao levar em consideração o estresse que a cultura sofreu dentro de uma escala de tempo (MENEGON et al. 2005).

Ademais, foi observado que a combinação do fungicida com o fertilizante, apresentou efeito antagônico, quer dizer, que o efeito dos produtos aplicados juntos é menor que a soma dos seus efeitos em aplicações isoladas (TREZZI et al. 2007). Segundo GAZZIERO (2015) relatou que o efeito antagônico em misturas, pode estar associado a alteração do pH da calda pela adição do fertilizante, afetando a eficácia do fungicida.

Em relação ao efeito residual dos tratamentos, de maneira geral os compostos isolados ou combinados, reduziram a AACPMM em $50 \%$ aos $30 \mathrm{DAE}$, quando comparado ao controle (Figura 1 e Figura 2). A maior eficácia do fungicida se atribui ao seu residual prologando devido a sua característica sistêmica de penetração e translocação no tecido vegetal (PICININI \& FERNANDES 2003). Segundo GOULART (1999), o período de proteção das plantas de trigo pelo tratamento de sementes com fungicida triadimenol, variou de 43 até 53 dias para o oídio e ferrugem da folha. 


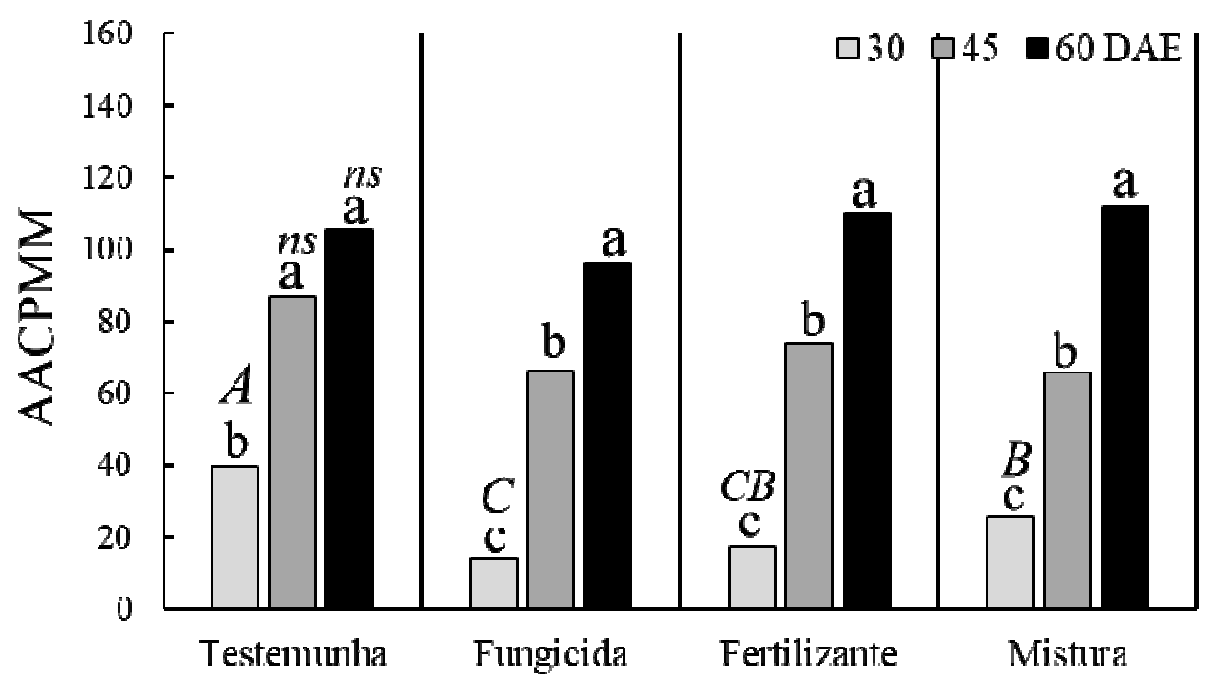

Figura 1. Área abaixo da curva de progresso da mancha marrom (AACPMM) em plantas de cevada com 30, 45 e 60 dias após a emergência (DAE), que receberam o tratamento de sementes com fungicida, fertilizante silicatado e a mistura fungicida + fertilizante silicatado e inoculação via foliar com Bipolaris sorokiniana. Letra minúscula, compara a eficiência de cada tratamento entre os DAE, pelo teste de Tukey $(p \leq 0,05)$. Letras maiúsculas, compara os tratamentos dentro de cada data de DAE, pelo teste de Tukey $(p \leq 0,05) . n s=$ não significativo.

Figure 1. Area under progress curve of the brown spot progress curve (AUCPBS) in barley plants 30,45 and 60 days after emergence (DAE), which received seed treatment with fungicide, silicate fertilizer and the fungicide + silicate fertilizer mixture, and leaf inoculation with Bipolaris sorokiniana. Lower case, compares the efficiency of each treatment among the DAE, by test Tukey test ( $p \leq 0.05)$. Capital letters, compare the treatments within each DAE date, by test Tukey $(p \leq 0.05)$. ns $=$ not significant.

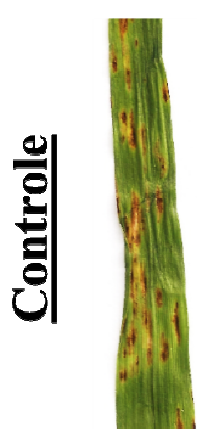

30

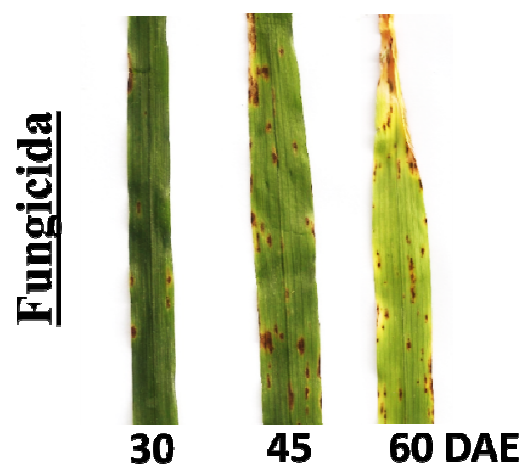

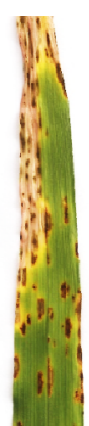

60 DAE

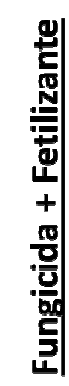

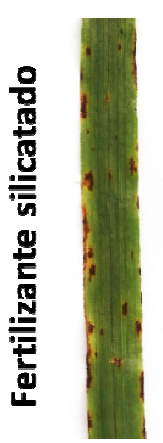

30

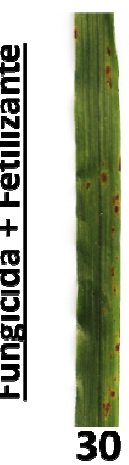

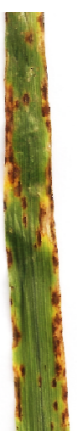

45

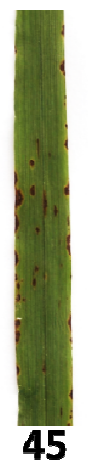

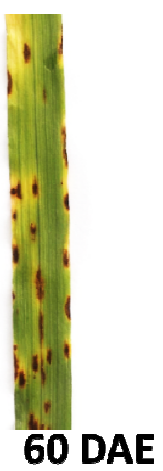

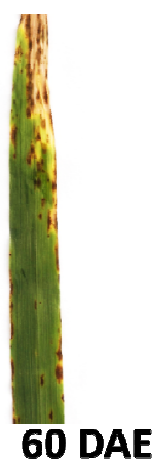

Figura 2. Severidade da mancha marrom em folhas de plantas de cevada com 30, 45 e 60 dias após emergência (DAE) que receberam o tratamento de sementes com fungicida, fertilizante silicatado e a mistura fungicida + fertilizante silicatado.

Figure 2. Severity of brown spot on leaves of barley plants 30, 45 and 60 days after emergence (DAE) that received seed treatment with fungicide, silicate fertilizer and the fungicide + silicate fertilizer mixture. 
Já em relação ao fertilizante silicatado, a sua ação está relacionada ao potencial do silício em ser absorvido pelo sistema radicular, promovendo uma barreira física formada pela sua polimerização na superfície foliar, bem como pelo estímulo nas defesas bioquímicas da planta (RODRIGUES \& DATNOFF 2015). Entretanto, não pode ser descartado que os demais elementos que compõem o fertilizante foliar também possam estar envolvidos na ativação de respostas de defesa da planta contra o patógeno.

Dessa forma, a proteção no estádio inicial da cultura da cevada pelo tratamento de semente pode conferir um retardamento no desenvolvimento da epidemia da mancha marrom, uma vez que reduziu a quantidade da doença e por conseguinte o inóculo secundário. Assim, conclui-se que o fertilizante silicatado e a mistura de fertilizante + fungicida reduzem a severidade da mancha marrom em plantas com até 30 dias, contudo com eficiência inferior ao fungicida isolado.

\section{AGRADECIMENTOS}

O presente trabalho foi realizado com apoio do CNPq (Conselho Nacional de Desenvolvimento Científico e Tecnológico) (Processo: 155829/2018-1). L. J. Dallagnol agradece ao CNPq pela bolsa de produtividade (Processo: 308149/2018-1).

\section{REFERÊNCIAS}

BRASIL. 2009. Manual de Análise Sanitária de Sementes. Ministério da Agricultura, Pecuária e Abastecimento. Secretaria de Defesa Agropecuária. Brasília: MAPA. 200p.

CAMILO GL et al. 2017. Qualidade fisiológica de sementes de soja durante o armazenamento após revestimento com agroquímicos. Revista de Ciências Agrárias 40: 180-189.

GAZZIERO DLP. 2015. Misturas de agrotóxicos em tanque nas propriedades agrícolas do Brasil. Planta Daninha 33: 8392.

GOULART ACP. 1999. Controle do oídio e da ferrugem da folha pelo tratamento de sementes de trigo com fungicidas. Dourados: Embrapa. 26p. (Boletim de Pesquisa).

LEMES E et al. 2019. Tratamento de sementes industrial: potencial de armazenamento de sementes de soja tratadas com diferentes produtos. Colloquium Agrariae 15: 94-103.

MACHADO BR \& QUEIROZ SEE. 2018. Efeito do tratamento de sementes de soja com silício e polímero na qualidade fisiológica das sementes e nas características agronômicas. Enciclopédia Biosfera 15: 1576-1584.

MENEGON AP et al. 2005. Expansão de lesão por manchas foliares em cevada e sua interação com a aplicação foliar de fungicidas. Fitopatologia Brasileira 30: 134-138.

PICININI EC \& FERNANDES JMC. 2003. Efeito do tratamento de sementes com fungicida sobre o controle de doenças na parte aérea do trigo. Fitopatologia Brasileira 28: 515-520.

PINTO NFJA. 2000. Tratamento fungicida de sementes de milho contra fungos do solo e o controle de Fusarium associado às sementes. Scientia Agricola 57: 483-486.

RODRIGUES FA \& DATNOFF LE (Eds). 2015. Silicon and Plant Disease. London: Elsevier. 145p.

SAATH KCO \& FACHINELLO AL. 2018. Crescimento da demanda mundial de alimentos e restrições do fator terra no Brasil. Revista de Economia e Sociologia Rural 56: 195-212.

TREZZI MM et al. 2007. Antagonismo das associações de clodinafop-propargyl com metsulfuron-methyl e 2,4-D no controle de azevém (Lolium multiflorum). Planta Daninha 25: 839-847.

TUNES LVM et al. 2014. Qualidade fisiológica, sanitária e enzimática de sementes de arroz irrigado recobertas com silício. Revista Ceres 61: 675-685. 